\title{
Musculoskeletal simulation model generation from MRI datasets and motion capture data
}

\author{
Jérôme Schmid ${ }^{1}$, Anders Sandholm², François Chung ${ }^{3}$, Daniel Thalmann ${ }^{4}$, \\ Hervé Delingette $^{5}$ and Nadia Magnenat-Thalmann ${ }^{6}$
}

\begin{abstract}
Today computer models and computer simulations of the musculoskeletal system are widely used to study the mechanisms behind human gait and its disorders. The common way of creating musculoskeletal models is to use a generic musculoskeletal model based on data derived from anatomical and biomechanical studies of cadaverous specimens. To adapt this generic model to a specific subject, the usual approach is to scale it. This scaling has been reported to introduce several errors because it does not always account for subject-specific anatomical differences. As a result, a novel semi-automatic workflow is proposed that creates subject-specific musculoskeletal models from Magnetic Resonance Imaging (MRI) datasets and motion capture data. Based on subject-specific medical data and a model-based automatic segmentation approach, an accurate modeling of the anatomy can be produced while avoiding the scaling operation. This anatomical model coupled with motion capture data, joint kinematics information and muscle-tendons actuators is finally used to create a subject-specific musculoskeletal model.
\end{abstract}

\footnotetext{
${ }^{1}$ University of Geneva, MIRALab

schmid@miralab.unige.ch

${ }^{2}$ Ecole Polytechnique Fédérale de Lausanne, Virtual Reality Laboratory anders.sandholm@epfl.com

${ }^{3}$ INRIA Sophia-Antipolis, Asclepios Research Team francois.chung@sophia.inria.fr

${ }^{4}$ Ecole Polytechnique Fédérale de Lausanne, Virtual Reality Laboratory daniel.thalmann@epfl.com

${ }^{5}$ INRIA Sophia-Antipolis, Asclepios Research Team

herve.delingette@sophia.inria.fr

${ }^{6}$ University of Geneva, MIRALab

thalmann@miralab.unige.ch
} 
4 Jérôme Schmid, Anders Sandholm, François Chung, Daniel Thalmann, Hervé Delingette and Nadia Magnenat-Thalmann

\section{Introduction}

Today neuromuscular simulation has proved to be a valuable tool in understanding human movements, from gait research [19], diagnostic and treatment of patients to teaching/training new physicians. During the last years, several simulation platforms have been developed, both commercial $[7,10]$ and open source based [9], along with several models with different level of details and complexity $[29,14]$.

The use of computer model/simulation has also allowed researchers and physicians to test what if scenarios, and even simulate different treatments before the physical treatment or surgery take place. A person gait cycle can be affected by many factors such as bone deformations [18] or static muscle contractors [25]. To simulate such patient with gait troubles or deformed skeleton, a full inspection of their anatomy should be first conducted. The most common neuromuscular models which are used today are all based on healthy, average-size adult male specimens [15]. Before the simulation of a person anatomy, the generic neuromuscular model has to be scaled to match the anatomy of the subject. The most two common ways are the isotropic scaling, where the whole model is scaled with the same scaling factor and the anisotropic scaling where each model part is scaled with an individual scaling factor. To determine these scaling factors, an initial motion capture is performed, where the subject stands still wearing 3D position markers placed at pre-defined anatomical positions. From these $3 \mathrm{D}$ markers, the length and position of each body segment can be computed to estimate the appropriate scaling factors to be applied on the generic musculoskeletal model. This scaling approach has several limitations and drawbacks. Firstly, the surface-based recordings cannot fully account for the large anatomical differences that exist among individuals. Secondly, they do not tackle the issue of skin displacement with respect to the underlying bones. A slight misplacement of a marker can generate large differences in the simulated model. In patient with cerebral palsy, it has been shown that the length of moment arms can significantly differ between a scaled and a subject-specific model [23].

The scaling is not the only issue; the complexity of the musculoskeletal system should be also addressed. Various studies tried to set up anatomical models at various levels of complexity. Scheepers et al. [22] and Aubel and Thalmann [2] described approaches based on anatomical concepts and constraints but the methods used unrealistic simplifications. One of the major limitations was that both studies were not subject-specific. More advanced models were presented like the works of Teran et al. [26] or Blemker and Delp [5] creating more complex anatomical models (e.g., fiber directions) to increase simulation accuracy. Although these studies relied on medical data sets offering the individualization of the modeling, they still require manual operations and corrections.

In this paper, a new framework that combines data from motion capture and segmented MRI images is introduced to create a subject musculoskeletal model 
and therefore remove the initial scaling step. The anatomical modeling pipeline uses first a stage of interactive modeling to create what is referred to as a generic anatomical model. This modeling needs to be performed only once and it considers anatomical constraints. Then the generic anatomical model is used in an automatic procedure that can be applied on any dataset to perform the individualization. The proposed approach can thus relieve the user from tedious interactive modelings. Finally, the anatomical model is combined with additional information produced from a reference motion capture frame to generate the complete musculoskeletal model in a semi-automatic fashion. If needed, a user interface is provided to alter/refine the full model. This model can be then used to simulate various motions.

\section{Musculoskeletal modeling pipeline}

When generating a musculoskeletal model, several sources of information and data have to be combined in a single simulation setup. The overall workflow, depicted in Fig. 1, is divided up into two phases, $a$ and $b$. In phase $a$, an individualized musculoskeletal model describing the subject is created, this model can be subsequently used in phase $b$ where a neuromuscular simulation is carried out to analyze a desired motion.

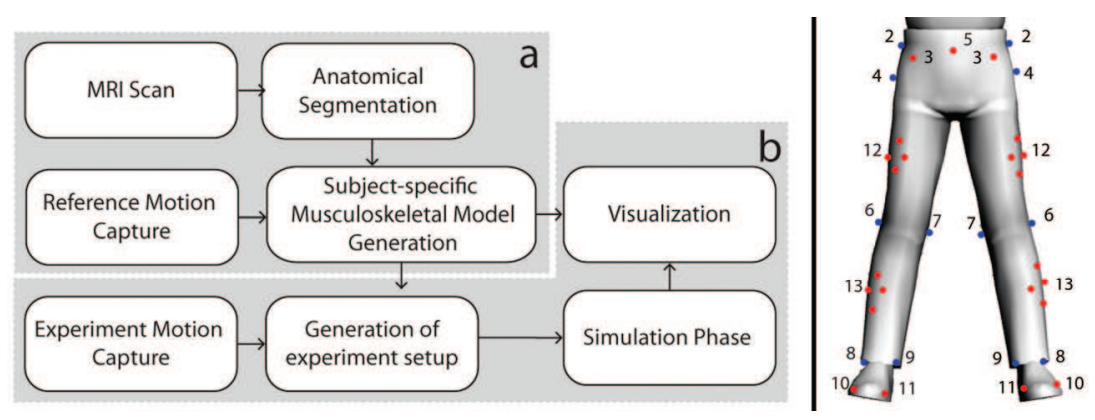

Fig. 1. Left: Workflow of subject-specific neuromuscular model generation and simulation. Phase $a$ generates the subject-specific neuromuscular model, in phase $b$ simulation and visualization are performed. Right: Reference (blue) and Skin (red) markers position overview, marker numbers correspond with Table 1. Only the lower limbs markers are shown.

In the first phase, a data acquisition process takes place. The lower limbs of the subject are scanned in a MRI device to produce datasets that are later on segmented (see corresponding section) in various anatomical components (e.g., muscles, bones). Motion capture sessions are also performed to collect information during a standing reference motion capture and one or several experiment motion captures. The later motion capture is coupled with synchronized EMG acquisitions and is used to simulate the motion that we want to study. By combining the 3D 
6 Jérôme Schmid, Anders Sandholm, François Chung, Daniel Thalmann, Hervé Delingette and Nadia Magnenat-Thalmann

models produced from the segmentation with the reference motion capture data, the subject-specific musculoskeletal model is generated. This model is then used in the second phase, along with the experiment motion capture data to simulate and analyze a specific motion.

In developing this framework, the OpenSim platform was chosen as the neuromuscular simulation engine, mainly because it is based on an open-source license and it supports several tools for model editing, fast simulation and advanced visualization features. OpenSim also supports a large collection of simulation possibilities such as inverse kinematics, muscular control and forward dynamics, whose output parameters include joint forces, muscle-induced acceleration and muscle power.

\section{Data acquisition}

\section{Markers setup for MRI and motion capture}

To create the musculoskeletal model, two marker sets are utilized. One set of skin markers is placed on muscle tissue while the other set of reference markers is placed on bone features, see Table 1 and right side of Fig. 1.

Table 1. Reference and skin markers.

\begin{tabular}{llll}
\hline & Placement & Type & Marker Set \\
\hline 1 & Acromioclavicular & Single Marker & Reference Marker \\
2 & Iliac crest & Single Marker & Reference Marker \\
3 & ASIS & Single Marker & Skin Marker \\
4 & Greater trochanter & Single Marker & Reference Marker \\
5 & Sacrum & Single Marker & Skin Marker \\
6 & Lateral knee & Single Marker & Reference Marker \\
7 & Medial knee & Single Marker & Reference Marker \\
8 & Lateral ankle & Single Marker & Reference Marker \\
9 & Medial ankle & Single Marker & Reference Marker \\
10 & $5^{\text {th }}$ metatarsal head & Single Marker & Skin Marker \\
11 & $1^{\text {st } \text { metatarsal head }}$ & Single Marker & Skin Marker \\
12 & Thigh & Cluster Set & Skin Marker \\
13 & Shank & Cluster Set & Skin Marker \\
\hline
\end{tabular}


The reference markers are located on easy-to-find bone features and are used both during the MRI acquisition and the motion capture. During motion capture, reflection markers are used, while markers filled with contrast agent are used during the MRI acquisition. The reference markers are used to put in correspondence the segmented and the motion capture data. Due to the fact that the reference markers are placed by hand at two different acquisitions, a small difference in placement can occur. However, this error is smaller than the error produced by skin movements during acquisition.

\section{MRI acquisition}

In close collaboration with radiologists, an adequate protocol for the imaging of soft and bony tissues was defined: Axial 2D T1 Turbo Spin Echo, TR/TE = $578 / 18 \mathrm{~ms}, \mathrm{FOV} / \mathrm{FA}=40 \mathrm{~cm} / 90^{\circ}$, matrix $/$ resolution $=512 \times 512 / 0.78 \times 0.78 \mathrm{~mm}$. To acquire a full lower limb, two different sessions took place and during each of them three acquisitions were performed. Each acquisition was based on the same protocol, but with varying slice thickness (e.g., higher thickness for long bones) to speed up the acquisition process. First session consisted in hip (thickness: $2 \mathrm{~mm}$ ), thigh $(10 \mathrm{~mm})$ and knee $(4 \mathrm{~mm})$; second session in knee $(2 \mathrm{~mm})$, leg $(10 \mathrm{~mm})$ and foot $(4 \mathrm{~mm})$. All acquisitions of a session were merged in a unique volume, and the two session volumes were registered together (thanks to an appropriate knee overlap). After cropping and resampling operations, two datasets have been created with the following size (resolution): $202 \times 398 \times 595(0.78 \times 0.78 \times 2.46 \mathrm{~mm})$ and $151 \times 213 \times 582(0.68 \times 0.68 \times 2.5 \mathrm{~mm})$ for the first and second datasets respectively. All acquisitions were performed at the University Hospital of Geneva on a 1.5T MRI device (Philips Medical Systems).

\section{Motion capture acquisition}

For the recording of the reference and experiment motion capture data, an optical motion capture system of eight ProReflex Qualisys (www.qualisys.com) video cameras was used. Prior to the session, reflective skin markers were fitted to the lower limbs according to Table 1 . Subjects were also equipped with electromyography (EMG) sensors from Noraxon (www.noraxon.com), to capture electrical activity in eight large lower limb muscles, see Table 2. During motion acquisition, two AMTI (www.amti.biz) force platforms were used to capture ground reaction force. Marker trajectories, EMG and force plate data were acquired, labeled and exported by using Qualisys Track Manager to C3D files. 
8 Jérôme Schmid, Anders Sandholm, François Chung, Daniel Thalmann, Hervé Delingette and Nadia Magnenat-Thalmann

Table 2. Electromyography electrodes placement.

\begin{tabular}{|c|c|c|}
\hline $\mathrm{Nr}$ & Muscle & \\
\hline 1 & Tibialis anterior & with additional ground marker \\
\hline 2 & Gastrocnemius & \\
\hline 3 & Vastus medialis & \\
\hline 4 & Soleus & \\
\hline 5 & Vastus lateralis & \\
\hline 6 & Rectus femoris & \\
\hline 7 & Biceps femoris & \\
\hline 8 & Gluteus maximus & \\
\hline
\end{tabular}

\section{Segmentation}

In order to model the various structures of interest with a high fidelity, a segmentation procedure is applied on medical datasets of the subject. The usage of manual segmentation is twofold. Firstly, it serves as a basis to automatic methods by providing (i) some prior knowledge (e.g., internal forces in deformable models that enforce the consistency with an a priori of a shape $[8,12]$ ) or (ii) training material (e.g., methods based on Principal Component Analysis (PCA) [6, 24] or classification methods like K-means [13] and Fuzzy C-means [4]). In general, more than one model is needed to produce satisfactory results. Secondly, it provides a ground truth for experiments. Segmentations generated by an automatic method can be thus compared with those obtained manually. In our case, manual segmentation has been performed by a medical expert, which allows us to validate it. In the following, our methodology for manual segmentation will be presented then a semi-automatic segmentation method will be depicted.

\section{Generic anatomical model construction}

\section{Manual delineation}

The manual delineation of the bones and muscles has been performed by a medical expert (see Fig. 2a). For each structure, a binary image was produced and then processed by the Marching Cubes algorithm [17] to generate 2-simplex meshes [8] representing the structures of interest. However, the generated meshes surface appeared not to be smooth enough. This is mostly explained by the dataset resolution (especially the large interslice distance) and the ubiquitous error made 
during a manual segmentation, which remains a subjective task. Ideally, the resolution should be as high as possible to provide detailed images and to avoid big jumps between consecutive MRI slices. This is not always feasible (e.g., device limitations, acquisition time restrictions). Furthermore, a lower resolution is preferred to reduce the memory footprint which results in a speed-up automatic algorithm. Since muscles are known to be anatomically smooth, the objective is to find a solution that makes them appear so. The technique we used consists first in refining the meshes and then in applying internal constraints on them. More precisely, we applied forces on the meshes so that their rigidity is the maximum. For that, we used the simplex angle continuity or $C_{2}$ constraint [8] used for shape recovery and which is defined as an average of the simplex angles at neighborhood vertices. Since we want to smooth our meshes in a large scale, we defined a great neighborhood size which intuitively corresponds to the notion of high rigidity for the mesh. The result was that most pits and bumps present on the meshes surface disappeared throughout the deformation process (see Fig. 2c). The smoothing based on $C_{2}$ constraint has also the advantage to reduce the shrinking effect obtained with standard Laplacian smoothing.

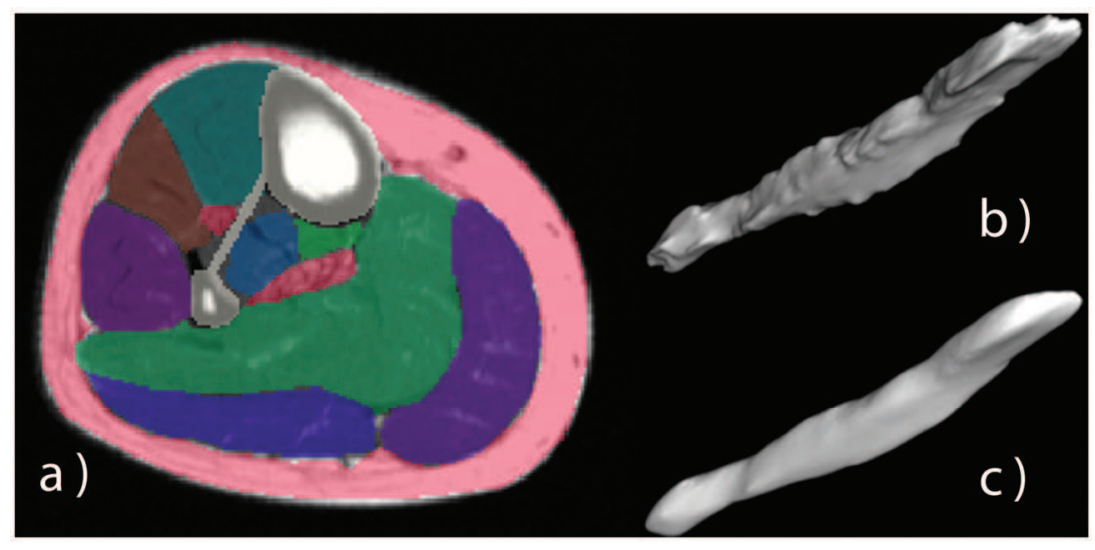

Fig. 2. a) Axial view of the leg with manual delineation in half transparency; b)-c) result of the smoothing procedure on the Flexor Digitorum Longus muscle: (b) mesh surface generated from the manual delineation and (c) mesh surface smoothed using the simplex angle continuity or $C_{2}$ constraint.

\section{Tendons}

Although the muscles are difficult to delineate manually, the tendons are easier to detect in the MRI. Indeed, they have a uniform appearance in the images (tendons appear as dark structures with respect to muscle tissue). An automatic 
10 Jérôme Schmid, Anders Sandholm, François Chung, Daniel Thalmann, Hervé Delingette and Nadia Magnenat-Thalmann

method based on maximum intensity ridge tracing $[3,20]$ was first used. Such method relies on the assumption that tendons are roughly tubular structures, which is especially the case for the leg tendons. These tracing methods have proved to be robust to noise. They provide also a simple way to model a structure as a series of centerline points (a position and a radius). Furthermore, such representation could be reused in another automatic method. However, this method may not always segment correctly or entirely the tendons because in some regions their intensity is corrupted by too strong artifacts (e.g., noise, adjacent structures, partial volume effect). As a result, tendons are difficult to follow through the slices. Moreover, some tendons are so close (e.g., fibularis longus and brevis tendons) that it is not possible to distinguish them without some prior knowledge.
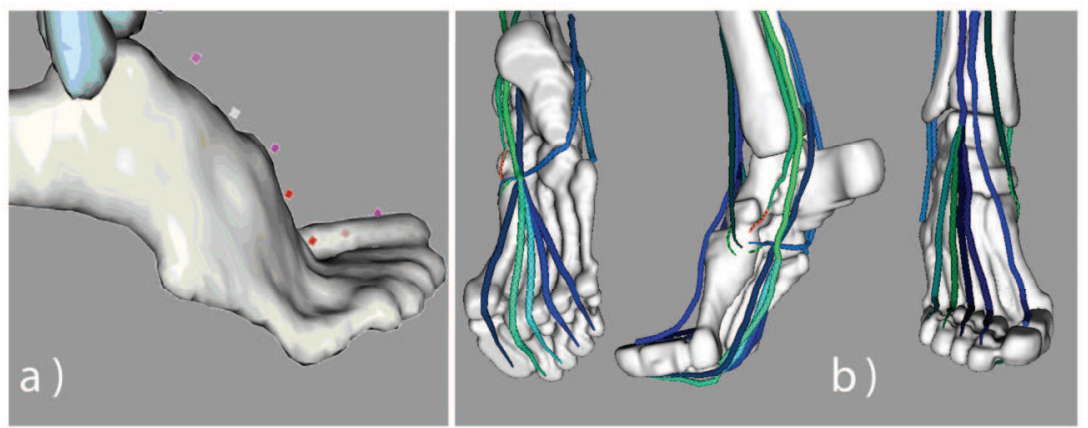

Fig. 3. Left: manual placement of centerline points for the Extensor Hallucis Longus tendon starting from the eponymous muscle until its attachment above the big toe. Right: results on various foot tendons.

To cope with these problems, missing centerline points were manually placed (Fig. 3a) on high curvature points of tendons using an editing tool, such as CardioViz3D [27]. Eventually, when the centerlines are complete (thanks to the automatic or manual modeling), simplex surfaces of tendons are automatically created. Fig. $3 \mathrm{~b}$ depicts some of the results for the foot tendons complex modeling.

\section{Attachments}

Anatomically, an attachment is defined as the linking region between two structures. For instance, tendons are usually attached to bones. The tendon attachments are thus defined as the tendon tissues sharing a common region with the bone tissues. Comparatively, it means that the meshes modeling these structures should be stuck together which is not the case a priori. Indeed, as accurate as the segmentation may be, the different generated meshes are not guaranteed to be attached together, especially after the smoothing procedure. Our solution is to manually place splines to define attachment regions as described in [11]. This 
provides a simple but efficient way to model attachment regions. Meshes are then deformed until they are stuck.

\section{Interpenetrations removal}

The smoothing and attachment procedures previously described can create non realistic interpenetrations between meshes. In order to remove them, a geometrical post-processing is applied. Let's consider the surfaces $S_{1}$ and $S_{2}$ in Fig. 4 that are interpenetrating each other. The aim is to move the colliding points of each surface, located in the penetration volume, to reach a non interpenetrating state.

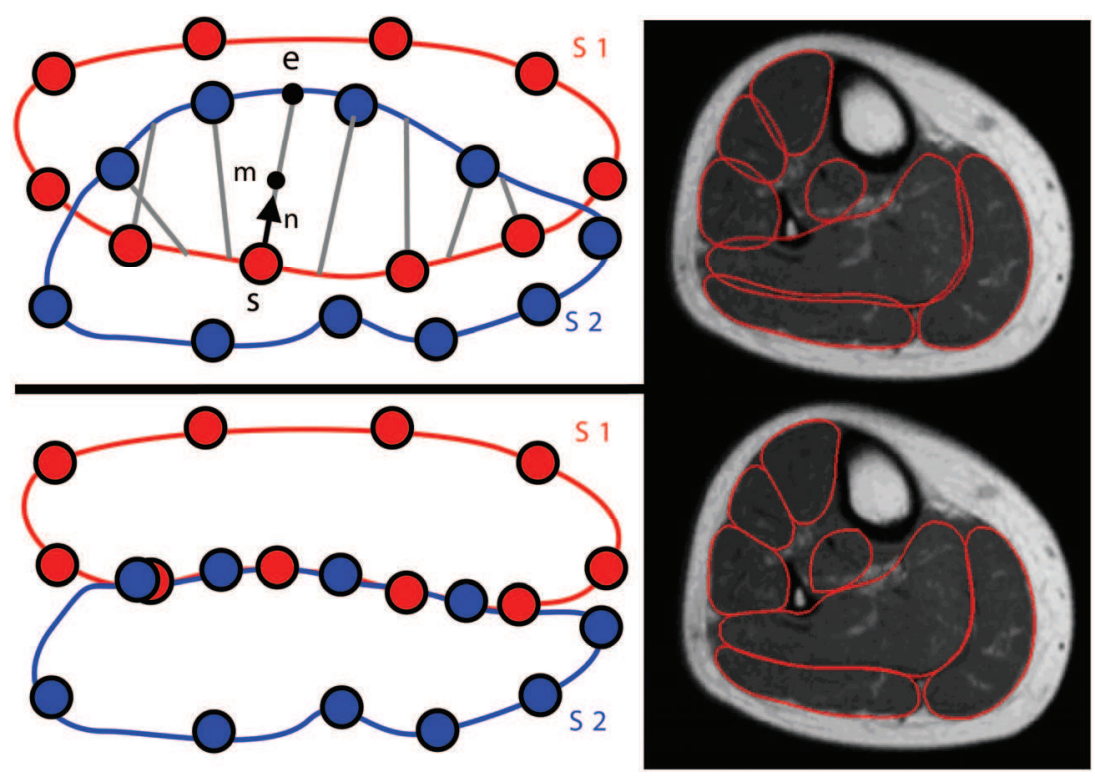

Fig. 4. Top-left: surfaces S1 and S2 are interpenetrating each other. Bottom-left: interpenetrations removal results. Right: results in real cases. It can be seen that muscles are not diffused through the neighbors anymore.

For a point $S \in S_{1}$ with inner normal $n$, the line passing through $S$ and directed by $n$ intersects $S_{2}$ in $e$. This point $e$ must be inside $S_{1}$ to be valid. The new position $m$ of the point $s$ is then chosen as:

$$
\left\{\begin{array}{l}
m=\{x \in[s, e] / f(m) \text { is minimal }\} \\
f(x)=\left(l_{1} * d_{1}(x)-l_{2} * d_{2}(x)\right)^{2}
\end{array}\right.
$$


12 Jérôme Schmid, Anders Sandholm, François Chung, Daniel Thalmann, Hervé Delingette and Nadia Magnenat-Thalmann

where $d_{i}(x)$ depicts the signed distance from $x$ to the surface $S_{i}$ and $l_{i}$ is a rigidity parameter. For instance, a configuration with $l_{1}<<l_{2}$ will lead to final points closer to surface $S_{2}$. This can be useful to resolve penetrations between a bone (very stiff material) and a muscle. The presented technique was inspired from [16], with the distinction that point $e$ is not defined as the projection of $s$ on $S_{2}$. Indeed, by using this projection, the point $m$ does usually not correspond to the new position of $s$ in the non colliding state. By using instead the normal direction $n$, the point $m$ will provide a better approximation. As a result, this technique avoids the usage of a resampling scheme as described in [16], points just need to be moved to their new positions. A perfect contact between the surfaces is nevertheless not ensured but the results are satisfactory when meshes resolution is not too low. Finally, implicit surfaces [1] are not used since the hypothesis of vicinity to surfaces may be invalid (i.e. in case of big interpenetrations). To speed up the process, precomputed signed distance maps can be used as well as the efficient golden section search technique [21] to minimize $f$.

\section{Semi-automatic segmentation}

The result of the previous section is a generic anatomical models collection of the various soft (muscles, tendons) and bony structures, from which various levels of details (LODs) are computed (4 for bones and 3 for soft structures). The objective now is to re-exploit these data as prior models in a semi-automatic method. This method could be then applied on any similar dataset coming from other subjects. The proposed method is mostly based on the deformable models method of Gilles et al. [12]. Mesh vertices are considered as lumped mass particles subjected to internal/external forces and evolving under the Newtonian law of motion.

Internal forces regulate shape evolution by enforcing constraints $[8,12]$ on the surface regularity (smoothness, curvature) and on the shape configuration. Shape constraints are simply enforced by predefining their simplex parameters. We added other priors [24] based on PCAs of training shapes and on the modeling of local deformations as a Gauss-Markov random process. External forces attract meshes towards anatomical boundaries by using image information (e.g., intensity, gradient) and impose non-penetration constraints. The attachments use mass modification methods to constrain vertices, while collision handling techniques prevent mesh penetrations through penalty forces.

The Newton equation relates forces to particle state. The resulting differential equations system is time discretized and solved by a stable implicit integration scheme [28]. The different mesh LODs are used in a multilevel framework using a force propagation mechanism. This strategy confers robustness and accuracy to the segmentation method. Conducted experiments $[12,24]$ reported an average ac- 
curacy of $1.5 \mathrm{~mm}$ for bone and muscle segmentation. The described segmentation procedure is equivalent to a Model to Image Registration. It is semi-automatic because generic anatomical models are initialized manually by placing landmarks. The power of using and registering generic shapes is that exact geometric correspondences are obtained (i.e., morphological features have the same vertex indexes across individual models, like attachments for instance).

\section{Anatomical modeling validation}

The proposed methods for correction (smoothing and interpenetrations removal) and the semi-automatic algorithms are still prone to errors. Indeed, they cannot always guarantee that the segmentation will perfectly delineate the structures of interest. To correct the most significant errors, a medical expert can define manually some constraints by placing points (e.g., points on the organ boundaries) in the MRI datasets. Forces are then applied on the meshes to account the expert constraints [11]. Similarly, forces based on image information (e.g., gradient) can be used to perform some local corrections. This approach gives nice results but as a general remark, one should avoid to place too many constraints or be tempted to blindly trust the image information. Indeed, the placement of constraints can present the same pitfalls as a manual delimitation: it is difficult to assess the organs transition between slices and ensure meshes smoothness. Consequently, it is essential to find a trade-off between reconstructed models quality and exact segmentation, since images cannot be totally trusted. Most importantly, the purpose of the segmentation is to provide models that must be reusable in our pipeline or in other studies.

\section{Musculoskeletal model generation}

The generation of a subject-specific musculoskeletal model can be divided into two parts. First, the anatomical segmented 3D objects have to be aligned with the reference motion capture data and secondly, the segmented objects have to be extended with additional information to create a complete musculoskeletal model.

\section{Data alignment}

The first step in generating a subject-specific musculoskeletal model is to align the segmented data with the motion capture reference frame, as depicted in the first step of the workflow (see Fig. 1). Doing this, the correct placement of the mo- 
14 Jérôme Schmid, Anders Sandholm, François Chung, Daniel Thalmann, Hervé Delingette and Nadia Magnenat-Thalmann

tion capture skin markers can be included into the model. This alignment is also performed to prepare the anatomical 3D objects for visualization purpose.

The alignment starts by reading the reference markers from the imported C3D file along with the reference markers from the segmented MRI images. For each bone segment (pelvis, femur and tibia), a rigid transformation matrix is calculated so that each segment can be correctly aligned with the motion capture data. To translate each bone segment, its corresponding markers are used, see Table 3. Currently, the marker extraction in the MRI images is performed manually but an automatic method could be achieved by using standard thresholding and mathematical morphology operators. The combination of various subject-specific data avoids performing any error-prone scaling.

By using each bone segment transformation, the skin markers defined in the reference motion capture file can be similarly expressed in the MRI coordinate space. For example, the skin marker Thigh (see Table 1) is transformed using the Femur matrices (see Table 3).

Table 3. Markers used to transform each bone segment.

\begin{tabular}{ll}
\hline Bone segment & Reference motion capture marker \\
\hline Pelvis & $\begin{array}{l}\text { left and right side markers of Iliac crest, } \\
\text { left and right side markers of Greater tro- } \\
\text { chanter }\end{array}$ \\
Femur & $\begin{array}{l}\text { Greater trochanter, Lateral knee, Medial } \\
\text { knee }\end{array}$ \\
Tibia & Lateral knee, Medial knee, Lateral ankle \\
& Medial ankle
\end{tabular}

\section{Model generation}

When the initial step of alignment is completed, the model consists of the 3D segmented objects and of motion capture markers. To generate a complete musculoskeletal model, information about muscles and joints has to be included. From the segmented 3D objects, each joint center is calculated. This joint center is used as the center point for the moment force generated by the muscles. Then for each joint, a kinematic function is defined to describe the kinematic behavior of each joint. By using this function and a given joint angle, the relative attachment point of the joints can be determined. Currently, this data is derived from Delp et al. [10]. To calculate the force that each muscle and its tendons can generate, each muscle is expressed into a set of one or more muscle-tendon actuators. These muscle-tendon actuators stretch between the muscles insertion points either as a straight line or via a wrapping point. At a wrapping point, the actuator changes direction to better describe the shape of the muscle as shown in Fig. 5. Wrapping points are also used to prevent muscle-tendon actuators to collide and enter a bone 
segment. If such a collision occurs, the wrapping becomes a fixed point and the actuator "wraps" around the bone, see Fig. 5b and 5c. The muscle-tendon actuator (Fig. 5a) is connected to each joint by using the tendon data from the segmentation process (see section "Attachments"), and the framework supports manual correction of both insertion and wrapping points. Currently, the description for all muscle-tendons and wrapping points are derived from Delp et al. [10] and OpenSim [9]. Eventually, the model is ready to be exported as a set of XML files in the OpenSim format.

a)

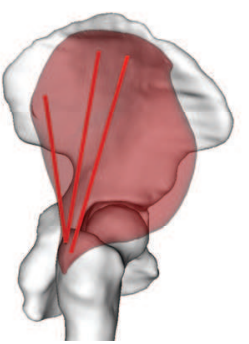

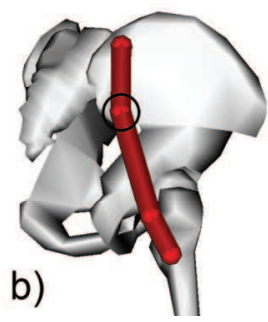

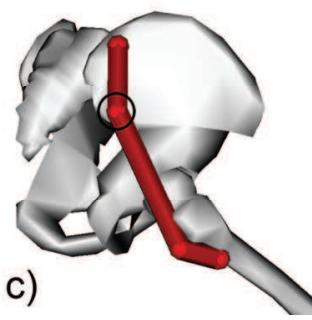

Fig. 5. a) Visualization of muscle-tendon actuators in Gluteus medius model; b-c) Muscle-tendon actuator representing Gluteus maximus; images $\mathrm{b}$ and $\mathrm{c}$ show the influence of wrapping points.

When a subject-specific simulation is carried out, the generated musculoskeletal model is loaded along with the experiment motion capture data. The model will now match the motion capture data, so no initial scaling has to be carried out. The only initial step before starting a simulation is to align each joint angle in the model with the corresponding joint angle in the first frame of the experiment motion capture. This is done using an inverse kinematic calculation which is performed by the OpenSim framework. The musculoskeletal model should now match the first frame of the motion capture data and the desired simulation can be executed.

\section{Results and discussion}

\section{Anatomical modeling results}

To illustrate the anatomical modeling, we applied our methodology on datasets produced from a female and healthy subject (age: 24 , height: $1 \mathrm{~m} 68$, weight: $58 \mathrm{~kg}$ ) acquisition. The institutional medical-ethical committee approved the study and the subject gave written informed consent. The manual modeling used to construct the generic anatomical model is applied on the leg area (knee to foot) while the 
16 Jérôme Schmid, Anders Sandholm, François Chung, Daniel Thalmann, Hervé Delingette and Nadia Magnenat-Thalmann

automatic method is performed on the hip-thigh-knee area. The automatic method relies on generic models of the thigh previously generated from a similar manual interactive method. Results are visible in Fig. 6a to $6 \mathrm{~d}$.

\section{Musculoskeletal modeling results}

Effort has been put in devising a framework to set up a musculoskeletal model from subject-specific data. Currently, this workflow has only been used on one subject, some results are visible in Fig. 6e. Therefore, no quantitative comparison between scaled and subject specific simulation is available. However, the initial step of scaling has been avoided and this should therefore remove a source of error in the musculoskeletal simulation. The proposed approach also supports the generation of musculoskeletal models from subject with no conventional bone structures (e.g., pathological bones), where scaling would not have been possible since the underlying bone structure can be hard to determine from the surface markers/measurements.
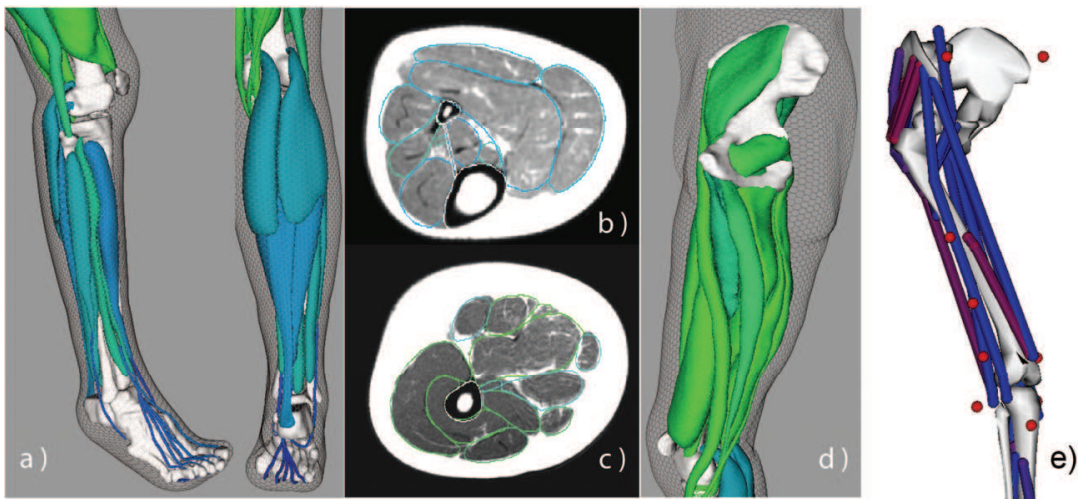

Fig. 6. Anatomical and musculoskeletal modeling results: a) Lateral and posterior view of the leg based on manual modeling; b) slice with segmentation contours overlay of the leg; c) slice with segmentation contours overlay of the thigh; d) lateral view of the thigh based on automatic segmentation; e) Musculoskeletal simulation in OpenSim showing bone surface, muscle-tendon actuators and motion capture markers.

\section{Conclusion and future work}

The workflow outlined in this paper allows the user to create a subject-specific musculoskeletal model based on MRI and motion capture acquisitions. The proc- 
ess is described as a semi-interactive approach where the segmentation is performed using a generic anatomical model. The segmented data is then extended with joint and muscular information so that a full musculoskeletal model is created. Currently, joint kinematics and tendon-muscular actuator information are derived from recognized literature. When the specific musculoskeletal model of the subject is generated, it can be used with any subject motion capture data to produce a desired simulation. The versatility of the framework is one of its qualities as it is not anymore necessary to rely on a standardized healthy male musculoskeletal model. It should be hence possible to simulate patients with disabilities as long as the acquired data reflects their pathology (e.g., organ shapes, abnormal EMG patterns, not conventional motions). Moreover, the framework being coupled with the OpenSim platform, a wide variety of measurements and simulation scenarios are available.

Yet a lot of work remains to fully validate the framework. Especially, more experiments must be carried out to compare the standard approach based on scaling and the proposed one. Accent will be put in studying more carefully the joint kinematics and the muscle-tendon actuators. The proposed workflow will have also to study, from a simulation viewpoint, the differences that exist among individuals such as gender, size and age. The approach will be then used in a medical context where some specific motions on pathological subjects will be examined. We hope that it will give some highlights into the mechanisms of pathologies and their treatments.

Acknowledgments This work is supported by the 3D Anatomical Human project (MRTN-CT2006-035763) funded by the European Union.

\section{References}

1. Alexa M, Adamson A (2004) On normals and projection operators for surfaces defined by pointsets. In: M. Alexa, M. Gross, H. Pfister, S. Rusinkiewicz (eds.) Proc. Eurographics Symposium on Point-based Graphics, pp. 149-156

2. Aubel A, Thalmann D (2001) Interactive modeling of the human musculature. In: HyeongSeok Ko (ed.) Proc. of Computer Animation, pp. 167-174

3. Aylward S, Bullitt E (2002) Initialization, noise, singularities, and scale in height ridge traversal for tubular object centerline extraction. IEEE Transactions on Medical Imaging 21(2): $61-75$

4. Bezdek J (1973) Fuzzy mathematics in pattern classification. Phd Thesis, Cornell University, Ithaca, NY

5. Blemker S, Delp S (2005) Three-dimensional representation of complex muscle architectures and geometries. Ann Biomed Eng 33:661-673

6. Cootes T, Hill A, Taylor C, Haslam J (1994) The use of active shape models for locating structures in medical images. Image and Vision Computing 12(6):355-366

7. Damsgaard M, Rasmussen J, Christensen ST, Surma E, de Zee M (2004) Analysis of musculoskeletal systems in the anybody modeling system. Simulation Modelling Practice and Theory $14(8): 1100-1111$ 
18 Jérôme Schmid, Anders Sandholm, François Chung, Daniel Thalmann, Hervé

Delingette and Nadia Magnenat-Thalmann

8. Delingette H (1999) General object reconstruction based on simplex meshes. Int. J. Comput. Vis. 32(2):111-146

9. Delp SL, Anderson FC, Arnold AS, Loan P, Habib A, John CT (2007) Opensim: Opensource software to create and analyze dynamic simulations of movement. IEEE Transactions on Biomedical Engineering 54(11):1940-1950

10. Delp SL, Loan J.P, Hoy MG, Zajac FE, Topp EL, Rosen JM (1990) An interactive graphicsbased model of the lower extremity to study orthopaedic surgical procedures. IEEE Transactions on Biomedical Engineering 37(8):757-167

11. Gilles B (2007) Anatomical and kinematical modelling of the musculoskeletal system from mri. Phd Thesis, University of Geneva

12. Gilles B, Moccozet L, Magnenat-Thalmann N (2006) Anatomical modelling of the musculoskeletal system from mri. In: R. Larsen, M. Nielsen, J. Sporring (eds.) MICCAI 2006. LNCS, vol. 4190, pp. 289-296

13. Hartigan J, Wong M (1979) A K-means clustering algorithm. J Roy Stat Soc C Appl Stat 28: $100-108$

14. Holzbaur K, Murray W, Delp S (2005) A model of the upper extremity for simulating musculoskeletal surgery and analyzing neuromuscular control. Annals of Biomedical Engineering 33(6):829-840

15. Horsman MK, Koopman H, van der Helm F, Prose L.P, Veeger H (2007) Morphological muscle and joint parameters for musculoskeletal modelling of the lower extremity. Clinical Biomechanics 22:239-247

16. Keiser R, Müller M, Heidelberger B, Teschner M, Gross M (2004) Contact handling for deformable point-based objects. In: B. Girod, M. Magnor, H.P. Seidel (eds.) Proc. of Vision, Modeling, Visualization '04 (VMV'04), pp. 315-322

17. Lorensen W, Cline H (1987) Marching cubes: A high resolution 3D surface construction algorithm. In: M.C. Stone (ed.) Proc. of the 14th annual conference on Computer graphics and interactive techniques, pp. 163-169. ACM New York, NY, USA

18. Metaxiotis D, Acclesa W, Siebela A, Doederleina L (2000) Hip deformities in walking patients with cerebral palsy. Gait \& Posture 11(2):86-91

19. Piazza S, Delp S (1996) The influence of muscles on knee flexion during the swing phase of gait. Journal of Biomechanics 29(6):723-733

20. Pock T, Janko C, Beichel R, Bischof H (2005) Multiscale medialness for robust segmentation of 3d tubular structures. In: A. Hanbury, H. Bischof (eds.) Proc. 10th Computer Vision Winter Workshop, pp. 93-102

21. Press WH, Flannery BP, Teukolosky SA, Vetterling WT (1992) Numerical Recipes in C, Second ed. Cambridge University Press

22. Scheepers F, Parent R, Carlson W, May S (1997) Anatomy-based modeling of the human musculature. In: SIGGRAPH'97, pp. 163-172

23. Scheysa L, Campenhoutc AV, Spaepenb A, Suetensa P, Jonkers I (2008) Personalized mrbased musculoskeletal models compared to rescaled generic models in the presence of increased femoral anteversion: effect on hip moment arm lengths. Gait \& Posture 28(3):358365

24. Schmid J, Magnenat-Thalmann N (2008) MRI bone segmentation using deformable models and shape priors. In: D. Metaxas, L. Axel, G. Szekely, G. Fichtinger (eds.) MICCAI 2008, Part I. LNCS, vol. 5241, pp. 119-126. Springer-Verlag Berlin Heidelberg

25. Tardieu G, Tardieu C (1987) Mechanical evaluation and conservative correction of limb joint contractures. Clinical Orthopaedics \& Related Research 219:63-69

26. Teran J, Sifakis E, Blemker S, Ng-Thow-Hing V, Lau C, Fedkiw R (2005) Creating and simulating skeletal muscle from the visible human data set. IEEE Trans Visual Comput Graph 11:317-328

27. Toussaint N, Mansi T, Delingette H, Ayache N, Sermesant M (2008) An Integrated Platform for Dynamic Cardiac Simulation and Image Processing: Application to Personalised Tetral- 
ogy of Fallot Simulation. In: Proc. Eurographics Workshop on Visual Computing for Biomedicine (VCBM)

28. Volino P, Magnenat-Thalmann N (2005) Implicit midpoint integration and adaptive damping for efficient cloth simulation. Comput Animation Virt World 16(3-4):163-175

29. Zhang LQ, Wang G (2001) Dynamic and static control of the human knee joint in abductionadduction. Journal of Biomechanics 34(9):1107-1115 
20 Jérôme Schmid, Anders Sandholm, François Chung, Daniel Thalmann, Hervé Delingette and Nadia Magnenat-Thalmann 